Physics

Physics Research Publications

\title{
Stoichiometry driven impurity configurations in compound semiconductors
}

G. Chen

S. Rodriguez
I. Miotkowski

A. K. Ramdas 


\title{
Stoichiometry Driven Impurity Configurations in Compound Semiconductors
}

\author{
G. Chen, I. Miotkowski, S. Rodriguez, and A. K. Ramdas \\ Department of Physics, Purdue University, West Lafayette, Indiana 47907, USA
}

(Received 26 October 2005; published 26 January 2006)

\begin{abstract}
Precise stoichiometry and departures therefrom in the composition of the tetrahedrally coordinated compound semiconductors allow impurity incorporation in more than one configuration. Ultrahigh resolution infrared spectroscopy of CdTe:O at low temperatures reveals a unique sharp doublet associated with the local vibrational modes of $\mathrm{O}_{\mathrm{Te}}$ in a $\left(\mathrm{O}_{\mathrm{Te}}-V_{\mathrm{Cd}}\right)$ complex with nearest neighbor Cd vacancy $V_{\mathrm{Cd}}$ and a single sharp line attributed to the local vibrational mode of $\mathrm{O}_{\mathrm{Te}}$ in a perfect CdTe. The uniaxial $\left(\mathrm{C}_{3 v}\right)$ symmetry of $\left(\mathrm{O}_{\mathrm{Te}}-V_{\mathrm{Cd}}\right)$ transforms to $T_{d}$ symmetry at $T^{*} \sim 300 \mathrm{~K}$, acquired due to an increasing rate of dynamic switching of the " $\mathrm{O}_{\mathrm{Te}}-V_{\mathrm{Cd}}$ " dangling bond in which the vacancy and its three next nearest neighbor $\mathrm{Cd}$ cations exchange positions as temperature $(T)$ approaches $T^{*}$; for $T \geq T^{*}$, the doublet thus transforms into a single, triply degenerate line.
\end{abstract}

DOI: 10.1103/PhysRevLett.96.035508

The physical issues underlying the structure, electronic levels, and vibrational excitations of point defects in semiconductors and the significant role they play in the design and performance of electronic and optoelectronic devices form an important area in current research on semiconductors. Spectroscopic signatures of point defects serve as insightful characterizations in basic studies and applications. In this context, localized vibrational modes (LVM) revealed in their infrared absorption spectra, for example, provide invaluable information into the nature of the defect as well as of the host [1,2].

The appearance of vacancies and interstitials originating from the lack of exact stoichiometry is a special feature of compound semiconductors. Cd vacancy in CdTe is a well documented example among the tetrahedrally coordinated binary semiconductors, where the more efficient incorporation of the anions than that of cations results in cation vacancies [3]. As an example of cation vacancy deliberately produced in a compound semiconductor, we cite Lee et al. [4], who studied optically detected magnetic resonance of $\mathrm{Zn}$ vacancies in $\mathrm{ZnSe}$ irradiated with $1.5 \mathrm{MeV}$ electrons. In this Letter, we report for the first time the striking manifestation of localized vibrational modes of oxygen impurities substitutionally incorporated into CdTe in which cation vacancies are either generated or suppressed deliberately by adopting specific crystal growth procedures. It transpires that, depending upon the crystal growth strategy, oxygen is bonded to three $\mathrm{Cd}$ nearest neighbors $(\mathrm{NN})$ and occurs near a $\mathrm{Cd}$ vacancy $V_{\mathrm{Cd}}$, where the fourth $\mathrm{Cd}$ would have been in a crystal with perfect stoichiometry; we refer to this complex as " $\mathrm{O}_{\mathrm{Te}}-V_{\mathrm{Cd}}$ " with $\mathrm{C}_{3 v}$ symmetry. In another crystal growth strategy, oxygen is indeed incorporated with the full complement of all four NN Cd's to which the $\mathrm{O}_{\mathrm{Te}}$ with $T_{d}$ symmetry is tetrahedrally bonded. These identifications have been made on the basis of their infrared signatures recorded with an ultrahigh resolution Fourier transform spectrometer. The two oxygen centers display unique infrared signatures which bear unmistakable imprints of their site symmetry.
PACS numbers: 61.72.Ji, 63.20.Pw, 78.30.Fs

An ultrahigh resolution Fourier transform infrared (FTIR) spectrometer capable of an ultimate resolution of $0.0026 \mathrm{~cm}^{-1}$, a HgCdTe-infrared detector for the $500-5000 \mathrm{~cm}^{-1}$ spectral range and a composite Si liquid helium bolometer for the $10-700 \mathrm{~cm}^{-1}$ range, and a variable temperature cryostat allowing measurements in the range of $1.8-300 \mathrm{~K}$ were employed to record the transmission spectra of CdTe doped with oxygen. The incident light is unpolarized due to the special features in the optical layout of the interferometer.

In Fig. 1, we display the absorption spectrum of CdTe in which oxygen has been introduced during the crystal growth by the addition of $\mathrm{CdO}$ to the starting material, the concentration of the nonstoichiometry-related $\mathrm{Cd}$ vacancies remaining close to that in pure CdTe. Postulating that oxygen replaces an isovalent Te anion in the vicinity of a Cd vacancy (see the model shown in Fig. 1), the defect center $\mathrm{O}_{\mathrm{Te}}-V_{\mathrm{Cd}}$ along with the three Cd's bonded to $\mathrm{O}_{\mathrm{Te}}$ will display local symmetry $\mathrm{C}_{3 v}$. The two extremely sharp infrared signatures, $\nu_{1}=1096.78 \mathrm{~cm}^{-1}$ and $\nu_{2}=$ $1108.35 \mathrm{~cm}^{-1}$ in the figure, recorded with the FTIR spectrometer set to $0.01 \mathrm{~cm}^{-1}$ resolution, have remarkably small full widths at half maximum (FWHM) of 0.165 and $0.137 \mathrm{~cm}^{-1}$, respectively; in addition, the intensity of $\nu_{2}$ is nearly twice that of $\nu_{1}$. The intensities of $\nu_{1}$ and $\nu_{2}$ increase with increasing $\mathrm{CdO}$. Another more successful crystal growth of CdTe for the incorporation of oxygen with $\mathrm{C}_{3 v}$ symmetry is one in which $\mathrm{TeO}_{2}$ is added to the $\mathrm{CdTe}$ charge prior to crystal growth. It can be seen that, for each $\mathrm{TeO}_{2}$ unit destroyed, two O's and one Te will be released. Te released in this process produces a corresponding additional number of vacancies. The intensities of $\nu_{1}$ and $\nu_{2}$ also increase with increasing $\mathrm{TeO}_{2}$. Thus, one can claim that $\nu_{1}$ and $\nu_{2}$ are oxygen-related LVM's associated with the $\left(\mathrm{O}_{\mathrm{Te}}-V_{\mathrm{Cd}}\right)$ centers. On the basis of their thermoelectric effect spectroscopy, together with firstprinciple band structure calculations of a defect in CdTe, Awadalla et al. [5] have obtained its ionization energy and attributed it to $\left(\mathrm{O}_{\mathrm{Te}}-V_{\mathrm{Cd}}\right)$. It would be of interest to estab- 


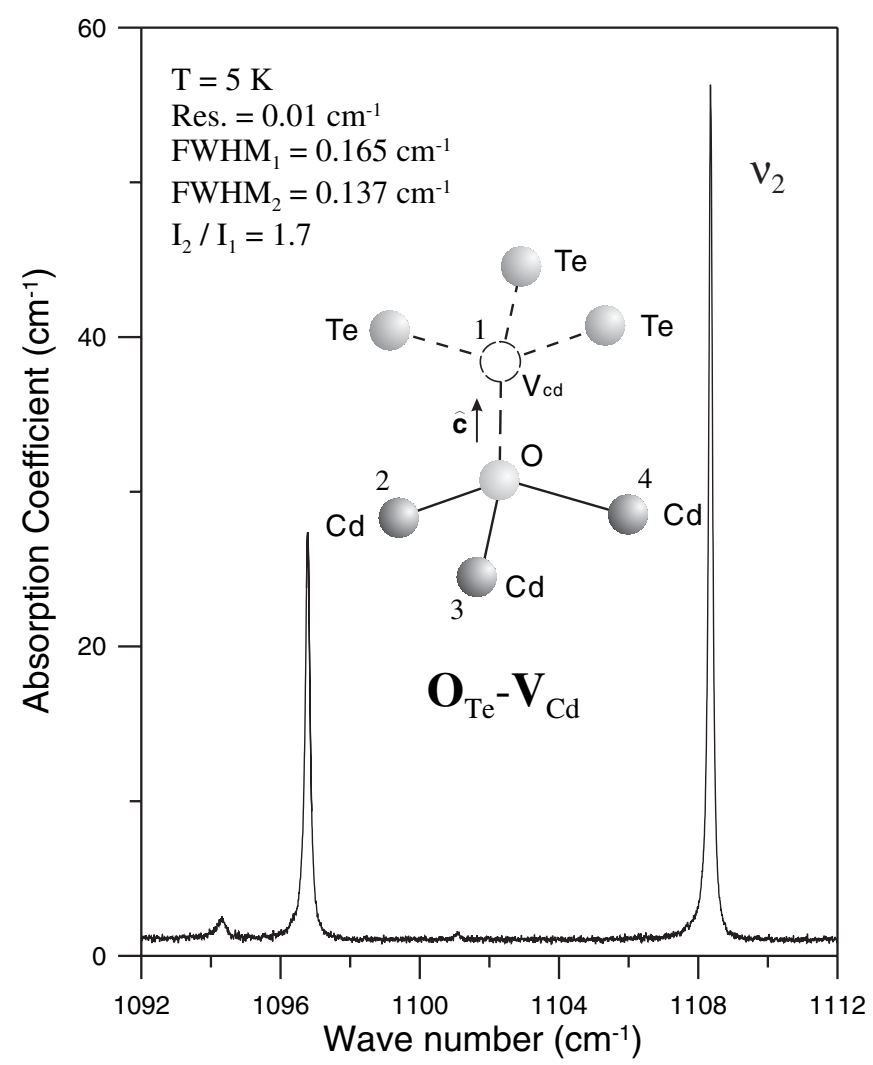

FIG. 1. The infrared absorption spectrum of the " $\mathrm{O}_{\mathrm{Te}}-V_{\mathrm{Cd}}$ " center with $\mathrm{C}_{3 v}$ symmetry in CdTe:O recorded at $5 \mathrm{~K}$ with a FTIR spectrometer set at $0.01 \mathrm{~cm}^{-1}$ resolution. The figure shows a model of substitutional oxygen replacing $\mathrm{Te}\left(\mathrm{O}_{\mathrm{Te}}\right)$, its three nearest neighbor $\mathrm{Cd}$ atoms, but with a vacancy $\left(V_{\mathrm{Cd}}\right)$ at the site of the fourth $\mathrm{Cd}$.

lish its relation to the spectroscopically observed $\left(\mathrm{O}_{\mathrm{Te}}-V_{\mathrm{Cd}}\right)$ defect with $\mathrm{C}_{3 v}$ symmetry in our investigation.

Each $\mathrm{O}_{\mathrm{Te}}-V_{\mathrm{Cd}}$ center is characterized by a $\hat{\mathbf{c}}$ axis along [111], [ $\overline{1} \overline{1} 1],[\overline{1} 1 \overline{1}]$, or [ $[1 \overline{1} \overline{1}]$. The two infrared active LVM's of $\mathrm{O}_{\mathrm{Te}}-V_{\mathrm{Cd}}$ (we are for the moment assuming the three NN Cd's in turn bonded to the rest of the crystal do not move) are given by group theory to be $\Gamma_{1}$ and $\Gamma_{3}$ species [6]; in the nondegenerate $\Gamma_{1}$ mode, oxygen vibrates along $\hat{\mathbf{c}}$, whereas in the doubly degenerate $\Gamma_{3}$, its motion is confined to the plane perpendicular to $\hat{\mathbf{c}}$. The $\Gamma_{1}$ mode responds to light polarized $\| \hat{\mathbf{c}}$, whereas the $\Gamma_{3}$ doublet couples to light polarized $\perp \hat{\mathbf{c}}$ [see Figs. 1 and 2(a)]. We note that the $\mathrm{O}_{\mathrm{Te}}-V_{\mathrm{Cd}}$ centers are distributed throughout the crystal with equal populations in each of the four directions of $\hat{\mathbf{c}}$, displaying thus an "orientational" degeneracy. It can also be shown that the polarization effects are averaged in such a fashion that no net polarization is expected for $\nu_{1}$ and $\nu_{2}$, independent of the direction of propagation, and the anisotropy of an individual center remains concealed (latent) in the otherwise cubic symmetry of the host [7]. The level ordering shown for the excited states for the fundamental of $\nu_{1}$ and $\nu_{2}$ shown as $\Gamma_{1}$ and $\Gamma_{3}$, respectively, is motivated by the latter being nearly twice as intense as

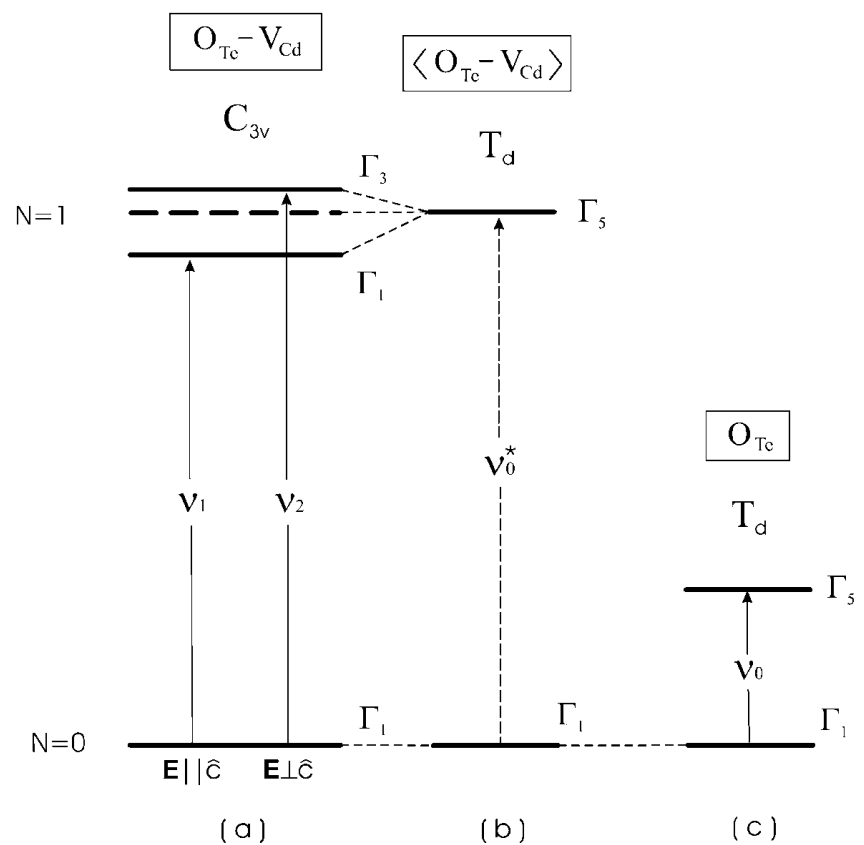

FIG. 2. Comparison of the schematic energy level diagrams and the fundamental dipole transitions in (a) " $\mathrm{O}_{\mathrm{Te}}-V_{\mathrm{Cd}}$ " center with $\mathrm{C}_{3 v}$ site symmetry, (b) " $\mathrm{O}_{\mathrm{Te}}-V_{\mathrm{Cd}}$ " with the dynamic switching of the dangling bond included, labeled as $\left\langle\mathrm{O}_{\mathrm{Te}}-V_{\mathrm{Cd}}\right\rangle$, and (c) " $\mathrm{O}_{\mathrm{Te}}$ " center with $T_{d}$ site symmetry.

the former. A stronger justification for the symmetry assignment will emerge from the experimental results, and their analysis presented below.

The temperature behavior of the $\nu_{1}$ and $\nu_{2}$ lines associated with the $\left(\mathrm{O}_{\mathrm{Te}}-V_{\mathrm{Cd}}\right)$ centers is shown in Fig. 3. It is remarkable that $\nu_{2}$ decreases with increasing temperature (as do all LVM frequencies generally, due to anharmonic coupling with phonons [8]), whereas $\nu_{1}$ actually increases; $\nu_{1}$ and $\nu_{2}$ eventually coalesce at $T^{*} \sim 300 \mathrm{~K}$, behaving for higher temperatures as a single mode of frequency $\nu_{0}^{*}$, which decreases as the temperature increases. What has happened is that two modes $\left[\nu_{1}\left(\Gamma_{1}\right)\right.$ and $\left.\nu_{2}\left(\Gamma_{3}\right)\right]$ of the $\mathrm{C}_{3 v}$ site symmetry of the $\left(\mathrm{O}_{\mathrm{Te}}-V_{\mathrm{Cd}}\right)$ center finally coalesce and become a single mode $\left[\nu_{0}^{*}\left(\Gamma_{5}\right)\right]$ of the same $\mathrm{O}_{\mathrm{Te}}-V_{\mathrm{Cd}}$ center at temperature around $T^{*}$, labeled as $\left\langle\mathrm{O}_{\mathrm{Te}}-V_{\mathrm{Cd}}\right\rangle$ in Fig. 2(b). We propose the hypothesis that the dynamic switching of the " $\mathrm{O}_{\mathrm{Te}}-V_{\mathrm{Cd}}$ " dangling bond among the four $\langle 111\rangle$ directions for $\hat{\mathbf{c}}$ occurs at an increasing rate; equivalently, one can view $V_{\mathrm{Cd}}$, originally at position 1 in the model shown in Fig. 1, occupying the positions denoted by 1, 2, 3, or 4 with equal probability for $T \geq T^{*}$ such that the center effectively assumes a $T_{d}$ site symmetry. On this basis, we interpret $\nu_{1}$ and $\nu_{2}$ to originate from the splitting of $\nu_{0}^{*}$ as a consequence of $\Gamma_{5}$ of $T_{d}$ decomposing into $\Gamma_{1}$ and $\Gamma_{3}$ of $\mathrm{C}_{3 v}$, the symmetry of $\left(\mathrm{O}_{\mathrm{Te}}-V_{\mathrm{Cd}}\right)$ at the lower temperatures. In the spirit of this splitting, viewed as resulting from a small perturbation, $\nu_{2}\left(\Gamma_{3}\right)$ is expected to be twice as intense as $\nu_{1}\left(\Gamma_{1}\right)$, the latter shifted twice as much below $\nu_{0}^{*}$ as $\nu_{2}\left(\Gamma_{3}\right)$ is above their weighted mean. Continuing this 


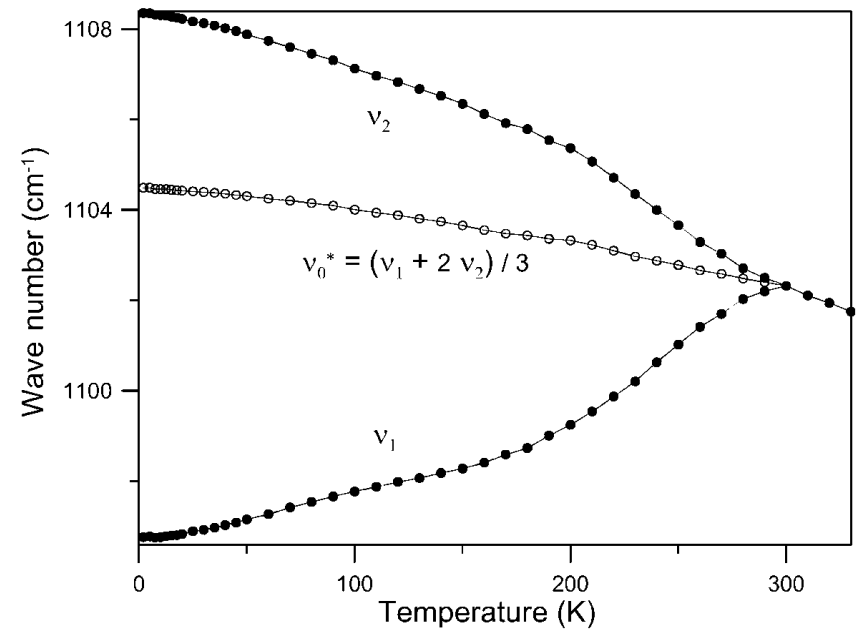

FIG. 3. Temperature dependence of $\nu_{1}$ and $\nu_{2}$ for the $\mathrm{O}_{\mathrm{Te}}-V_{\mathrm{Cd}}$ center from 2 to $330 \mathrm{~K}$ (solid circles) and the weighted average of $\nu_{1}$ and $\nu_{2}$, i.e., $\nu_{0}^{*}=\left(\nu_{1}+2 \nu_{2}\right) / 3$, calculated for each temperature (open circles).

line of argument, one can deduce $\nu_{0}^{*}=\left(\nu_{1}+2 \nu_{2}\right) / 3$ for each temperature as shown in Fig. 3. What is particularly impressive is that $\nu_{0}^{*}$ derived in this manner melds smoothly with that experimentally observed above $T^{*}$. Experiments performed with decreasing temperature show a complete restoration of the $\left(\nu_{1}, \nu_{2}\right)$ doublet below $T^{*}$, demonstrating that $\left(\nu_{1}, \nu_{2}\right)$ below and $\nu_{0}^{*}$ above $T^{*}$ are completely interconvertible.

The remarkable decrease of $\left(\nu_{2}-\nu_{1}\right)$, from $11.57 \mathrm{~cm}^{-1}$ at $2 \mathrm{~K}$ to zero at $T^{*}$, is a type of temperature behavior of LVM's of defect center not reported in the literature to the best of our knowledge. If one assumes that the temperature dependence of $\left(\nu_{2}-\nu_{1}\right)$ is given by $\left(\nu_{2}-\nu_{1}\right)_{T}=\left(\nu_{2}-\right.$ $\left.\nu_{1}\right)_{0}[1-\exp (-W / k T)],\left(\nu_{2}-\nu_{1}\right)_{T}$ and $\left(\nu_{2}-\nu_{1}\right)_{0}$ being the separations at $T$ and $0 \mathrm{~K}$, respectively, and $W$, a single activation energy for the underlying process, a plot of $\ln \left[1-\left(\nu_{2}-\nu_{1}\right)_{T} /\left(\nu_{2}-\nu_{1}\right)_{0}\right]$ vs $T^{-1}$ did not yield a straight line as expected but rather a curve resembling Fig. 3 in Fukai and Sugimoto [9], presumably associated with the multiple steps necessary for the dangling bond to switch from one $\hat{\mathbf{c}}$ axis to another. It is relevant to note that, in their electron paramagnetic resonance experiments on the vacancy trapped next to substitutional $\mathrm{P}$ (Si- $E$ center) in electron irradiated phosphorus doped $\mathrm{Si}$, Watkins and Corbett [10] discovered motional effects at temperatures as low as $250-300 \mathrm{~K}$ associated with the destruction and reconstitution of the bonds between $\mathrm{Si}$ atoms which are next nearest neighbors. As other examples of uniaxial defects with orientational degeneracy acquiring higher symmetry, we cite the extensive studies of Haller and coworkers on uniaxial defects in Ge [11,12] and the work of Muro and Sievers in Si [13], who have invoked "dynamic tunneling" as the microscopic process. The $\mathrm{O}_{\mathrm{Te}}-V_{\mathrm{Cd}}$ center in CdTe is a unique example of a trigonal defect with orientational degeneracy in a cubic crystal acquiring the higher $T_{d}$ symmetry by a dynamic switching of bonds as the microscopic process and revealed in their LVM's and their temperature dependence. The increasing width of $\nu_{1}$ and $\nu_{2}$ and the decrease of $\nu_{0}^{*}$ as a function of temperature follows a behavior pattern not unlike that of the LVM of $\mathrm{H}^{-}$in $\mathrm{CaF}_{2}$ by Elliott et al. [8] and attributed to anharmonic interactions with the host lattice vibrations.

It is of fundamental interest to enquire if oxygen can be indeed substitutionally incorporated into CdTe with the full complement of four NN Cd's and, hence, with $T_{d}$ symmetry. In order to accomplish this, we grew CdTe with the addition of $\mathrm{CdO}$ but with excess $\mathrm{Cd}$ to provide $\mathrm{O}$, on the one hand, and to suppress vacancies, on the other, and thus create conditions conducive to the formation of centers of oxygen with four Cd's surrounding it. From the knowledge of the LVM of $\mathrm{S}_{\mathrm{Te}}$ in CdTe [2], we estimated the spectral range in which to explore the occurrence of the LVM of $\mathrm{O}_{\mathrm{Te}}$ to be between 325 and $375 \mathrm{~cm}^{-1}$. As can be seen in Fig. 4, we indeed discovered the single infrared active mode of such a center $\left(\nu_{0}\right)$ occurring at $349.79 \mathrm{~cm}^{-1}$. We confirmed this assignment from the unmistakable increase in its intensity with increasing $\mathrm{CdO} ; \nu_{0}$ is then the infrared active triply degenerate mode $\Gamma_{1} \rightarrow \Gamma_{5}$ shown in Fig. 2(c). In such specimens, $\nu_{1}$ and $\nu_{2}$ are absent or occur

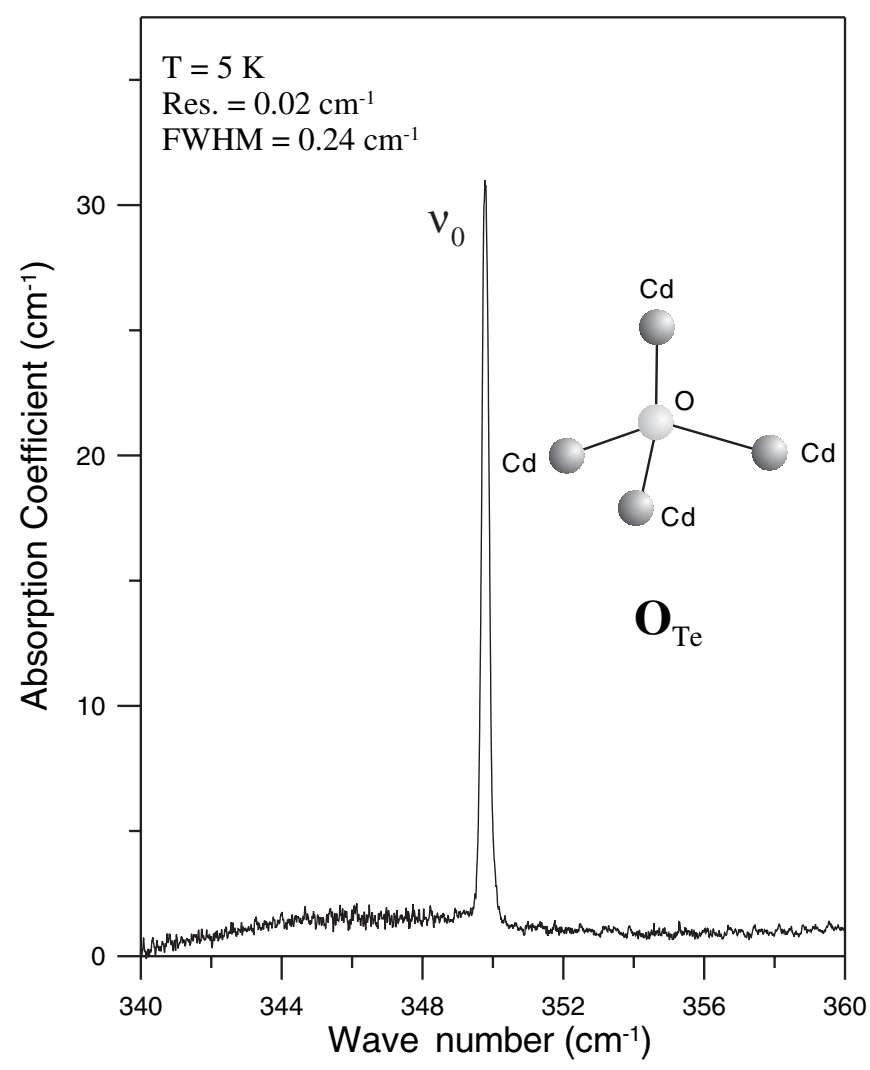

FIG. 4. The infrared absorption spectrum of the " $\mathrm{O}_{\mathrm{Te}}$ " center with $T_{d}$ symmetry in CdTe:O recorded at $5 \mathrm{~K}$ with a FTIR spectrometer set at $0.02 \mathrm{~cm}^{-1}$ resolution. Also shown is the model of $\mathrm{O}_{\mathrm{Te}}$ along with its four nearest neighbor $\mathrm{Cd}$ atoms. 
with extremely low intensities, whereas in specimens designed for observing the $\left(\nu_{1}, \nu_{2}\right)$ doublet, $\nu_{0}$ is conspicuous by its absence. We also note that the FWHM's are noticeably larger (at $300 \mathrm{~K}$ nearly twice) for $\nu_{1}$ and $\nu_{2}$ in comparison to that of $\nu_{0}$. This difference could well be attributed to the additional dynamic switching associated with $\nu_{1}$ and $\nu_{2}$. Neither $\nu_{1}$ and $\nu_{2}$ nor $\nu_{0}$ exhibit, even under the highest resolution and at the lowest temperature, the "host isotopic effect" shown by $\mathrm{Mg}^{2+}$ in CdTe [2], presumably due to the significantly lighter oxygen relative to $\mathrm{Cd}$.

The weighted average of $\nu_{1}$ and $\nu_{2}$, i.e., $\left(\nu_{1}+2 \nu_{2}\right) / 3=$ $\nu_{0}^{*}$ at $5 \mathrm{~K}$ is $1104.49 \mathrm{~cm}^{-1}$, is more than threefold higher than $\nu_{0}$ at $349.79 \mathrm{~cm}^{-1}$. We attribute this to a significant shortening of the Cd-O bonds in the " $\mathrm{O}_{\mathrm{Te}}-V_{\mathrm{Cd}}$ " centers, followed by the lifting of the threefold degeneracy of $\Gamma_{5}$ in the $T_{d}$ site symmetry, and transforming it into a nondegenerate and a doubly degenerate mode of $\mathrm{C}_{3 v}$ as reflected in Fig. 2. In order to estimate the effect of the decrease in the Cd-O bond length, we carried out a calculation based on the following assumptions: (1) The $\mathrm{Cd}$ atoms, bonded to $\mathrm{O}_{\mathrm{Te}}$ with or without a vacancy in its vicinity, remain fixed due to their significantly larger atomic mass. (2) The interaction between $\mathrm{Cd}$ and $\mathrm{O}$ is described by a potential associated with changes in bond stretching and bond bending. Although the model shows that $\nu_{1}$ and $\nu_{2}$ are larger than $\nu_{0}$ and $\nu_{2}>\nu_{1}$, the values of the three are comparable. We conclude that perturbation of the $\mathrm{Cd}-\mathrm{O}$ bond lengths and bond angles in $\left(\mathrm{O}_{\mathrm{Te}}-V_{\mathrm{Cd}}\right)$ with respect to those for $\mathrm{O}_{\mathrm{Te}}$ is too significant to be treated as small in order for $\nu_{1}$ and $\nu_{2}$ to be $\sim$ threefold larger than $\nu_{0}$. In this context, we draw attention to the fact that, in the $\left(\mathrm{O}_{\mathrm{Te}}-V_{\mathrm{Cd}}\right)$ center, the dangling bond pointing from $\mathrm{O}_{\mathrm{Te}}$ towards the absent $\mathrm{Cd}$ cation together with the other three $\mathrm{O}_{\mathrm{Te}}-V_{\mathrm{Cd}}$ dangling bonds (model in Fig. 1) define $V_{\mathrm{Cd}}$. We therefore speculate that the charge cloud associated with $V_{\mathrm{Cd}}$ may be redistributed into the $\mathrm{Cd}-\mathrm{O}$ bonds, thereby significantly increasing the effective force constants for $\nu_{1}$ and $\nu_{2}$. We qualitatively reflect this in Figs. 2(b) and 2(c), indicative of $\nu_{0}^{*}>\nu_{0}$ by a factor of 3 .

The work reported in this Letter demonstrates that the study of local vibrational modes provides unique insights in the context of the environment in which a point defect finds itself in a host. The observation of the LVM's of substitutional oxygen in the CdTe host as " $\mathrm{O}_{\mathrm{Te}}$ " as well as " $\mathrm{O}_{\mathrm{Te}}-V_{\mathrm{Cd}}$ "; the higher $T_{d}$ symmetry acquired by $\left(\mathrm{O}_{\mathrm{Te}}-V_{\mathrm{Cd}}\right)$ as a consequence of the rapid switching of the dangling bond among the four $\langle 111\rangle$ directions around a given $\mathrm{O}_{\mathrm{Te}}$ with increasing temperature are the highlights of the present work. Thanks to the low temperature sharpness of their spectroscopic signatures, on the one hand, and the essential simplicity of the underlying theoretical consider- ations, on the other, the studies of LVM in semiconductors have led to (a) discovery of well resolved lines according to the isotopic abundance of the impurity, (b) characterization of disorder in a multinary alloy, (c) the host isotopic fine structure [2], and (d) in this Letter, the unique features associated with perfect stoichiometry and departures therefrom. The present work underscores the important role stoichiometry plays in the impurity configurations in compound semiconductors. On the basis of the simplicity of the two impurity configurations of oxygen in CdTe, achieved reproducibly with controlled growth procedures, it is of interest to enquire if such defects might also occur in many other tetrahedrally coordinated compound semiconductors. In their search, suitable crystal growth strategies and infrared spectroscopy (as in the present case), Raman spectroscopy, and a variety of magnetic resonance techniques can be expected to play a fruitful role.

The authors acknowledge support from the National Science Foundation (DMR 0405082) and Purdue University for an Academic Reinvestment grant.

[1] A.S. Barker and A. J. Sievers, Rev. Mod. Phys. 47, Suppl. 2, S1 (1975).

[2] M. D. Sciacca, A. J. Mayur, N. Shin, I. Miotkowski, A. K. Ramdas, and S. Rodriguez, Phys. Rev. B 51, 6971 (1995); M. D. Sciacca, A. J. Mayur, H. Kim, I. Miotkowski, A. K. Ramdas, and S. Rodriguez, Phys. Rev. B 53, 12878 (1996).

[3] See, for example, P. Rudolph, Prog. Cryst. Growth Charact. Mater. 29, 275 (1994).

[4] K. M. Lee, Le Si Dang, and G. D. Watkins, Solid State Commun. 35, 527 (1980).

[5] S. A. Awadalla, A. W. Hunt, K. G. Lynn, H. Glass, C. Szeles, and S.H. Wei, Phys. Rev. B 69, 075210 (2004).

[6] We use the notation for the irreducible representation in G. F. Koster, J. O. Dimmock, R. G. Wheeler, and H. Statz, Properties of the Thirty-Two Point Groups (MIT, Cambridge, MA, 1963).

[7] A. A. Kaplyanskii, Opt. Spectrosc. (USSR) 16, 329 (1964).

[8] R. J. Elliott, W. Hayes, G. D. Jones, H. F. Macdonald, and C. T. Sennett, Proc. R. Soc. A 289, 1 (1965).

[9] Y. Fukai and H. Sugimoto, Adv. Phys. 34, 263 (1985).

[10] G. D. Watkins and J. W. Corbett, Phys. Rev. 134, A1359 (1964).

[11] E. E. Haller and L. M. Falicov, Phys. Rev. Lett. 41, 1192 (1978); E. E. Haller, B. Joós, and L. M. Falicov, Phys. Rev. B 21, 4729 (1980).

[12] B. Joós, E. E. Haller, and L. M. Falicov, Phys. Rev. B 22, 832 (1980); J. M. Kahn, R. E. McMurray, E. E. Haller, and L. M. Falicov, Phys. Rev. B 36, 8001 (1987).

[13] K. Muro and A. J. Sievers, Phys. Rev. Lett. 57, 897 (1986). 